\title{
Un sistema de modelamiento para evaluar las consecuencias económicas del cambio climático en el Caribe
}

\author{
Roberto Roson
}

RESUMEN

El Climate Impacts Assessment Model de la Comisión Económica para América Latina y el Caribe (ECLAC-CIAM) es una plataforma de modelado creada para evaluar las consecuencias económicas del cambio climático en el Caribe, que puede consultarse, descargarse e incluso modificarse libremente. La versión disponible es un modelo completamente desarrollado que puede utilizarse con facilidad para efectuar ejercicios de simulación. En este trabajo se realiza una descripción general del modelo y se presenta un ejercicio de simulación a modo de ejemplo. En los resultados del ejercicio se destaca que el Caribe es una región muy vulnerable en la que el cambio climático producirá consecuencias económicas considerables y negativas. 


\section{I}

\section{Introducción}

El cambio climático es un fenómeno muy complejo. Para estudiarlo, se utilizan modelos informáticos a gran escala con los que se simulan condiciones climáticas futuras de acuerdo con diferentes hipótesis y suposiciones. Las consecuencias del cambio climático y los costos y beneficios de las políticas de mitigación y adaptación se evalúan mediante modelos socioeconómicos. Con frecuencia, los modelos físicos y sociales se combinan en complejos modelos de evaluación integrada.

Una conclusión que se repite en numerosos análisis y estudios es que las consecuencias negativas del cambio climático afectarán sobre todo a los países en desarrollo de América Latina y el Caribe, África y Asia. Esto plantea un problema de equidad en las negociaciones internacionales, como las de la Convención Marco de las Naciones Unidas sobre el Cambio Climático, pues históricamente los países en desarrollo han sido responsables de una parte insignificante de las emisiones de gases de efecto invernadero. Los modelos cuantitativos pueden ser de gran utilidad para determinar las cargas y los beneficios de las políticas mundiales sobre el clima.

Lamentablemente, las regiones más vulnerables son también aquellas donde es más difícil realizar un análisis de evaluación riguroso debido a la falta de datos y competencia profesional. En el Caribe, por ejemplo, se careció durante mucho tiempo de una herramienta para evaluar las consecuencias sociales del cambio climático. En general, no existen modelos socioeconómicos cuantitativos centrados en la región caribeña y el uso de modelos de simulación, optimización, econométricos y de previsión no se ha difundido en el área, ni siquiera en instituciones como los bancos centrales nacionales (con pocas excepciones, entre ellas Jamaica). Por ese motivo, las bases de datos necesarias para respaldar e implementar modelos aplicados son inexistentes o inadecuadas y los responsables de la formulación de políticas generalmente ignoran la existencia de modelos económicos cuantitativos y sistemas de apoyo a las decisiones.

Este problema se afrontó recientemente en la sede subregional de la CEPAL para el Caribe, con el respaldo financiero de la Agencia de Australia para el Desarrollo Internacional. En la CEPAL se encargó un estudio con el objetivo de crear un modelo de prototipo o modificar un sistema ya existente para analizar las consecuencias del cambio climático en el Caribe en un sistema de modelado. En este trabajo se describe el modelo creado con ese propósito, el ECLAC-Climate Impacts Assessment Model (CIAM), y se presenta un ejercicio de simulación a modo de ejemplo.

El modelo ECLAC-CIAM se ha concebido y desarrollado sobre la base de algunos conceptos fundamentales. En primer lugar, se ha seguido un enfoque sistémico respecto del modelado de los efectos climáticos, pues el cambio del clima es un fenómeno universal y la economía mundial se ha globalizado. Por ejemplo, los efectos económicos del cambio climático en el Caribe pueden obedecer más a las repercusiones físicas registradas en los Estados Unidos de América que al impacto físico directo en la propia región. No obstante, en la mayoría de los estudios sectoriales no se tiene presente ese tipo de relaciones sistémicas.

En segundo lugar, el modelo permite la consideración de múltiples efectos en forma simultánea, pues el cambio climático produce diferentes consecuencias económicas en una gran variedad de sectores. Además del aumento del nivel del mar, se pueden mencionar, entre otros, los efectos en la productividad agrícola (debido a cambios en las precipitaciones, a una mayor evapotranspiración y a la fertilización carbónica), la demanda de turismo (cambios en los ingresos, los precios y el atractivo del turismo), la demanda de energía (incremento de la demanda de refrigeración, reducción de la demanda de calefacción), y en la salud humana y la productividad del trabajo (variaciones en la mortalidad y la morbilidad). Desde el punto de vista de la formulación de políticas, la evaluación simultánea de varios efectos es necesaria porque con frecuencia se debe: i)evaluar el efecto general del cambio climático en un país o una región en particular; ii) conocer la proporción de cada efecto sectorial puede ayudar a determinar las prioridades de las políticas de adaptación, y iii) algunos efectos pueden neutralizarse entre sí.

En tercer lugar, el modelo se ha diseñado con una estructura modular de fácil acceso y comprensión, de modo que otros investigadores, posiblemente caribeños, puedan introducir fácilmente nuevos elementos para acompañar los avances en el campo de la ciencia climática. De hecho, se espera producir un "efecto multiplicador" y que el ECLAC-CIAM sirva para promover el desarrollo de otras investigaciones y modelos. 
Una de las principales dificultades de la simulación con modelos matemáticos complejos es que puede requerir un programa de computación específico y costoso, que los científicos de los países en desarrollo tal vez no puedan permitirse. Se intentó superar este obstáculo basando el ECLAC-CIAM exclusivamente en programas auxiliares que pueden descargarse y utilizarse sin costo. Los códigos, datos y parámetros del programa, así como la documentación y los enlaces al programa computacional auxiliar, están disponibles gratuitamente en http://venus.unive.it/roson/ciam.html.
El presente trabajo se divide en cinco secciones incluida esta Introducción. Mientras que en la segunda sección se realiza un breve análisis del estado de la técnica de los modelos numéricos disponibles para evaluar los efectos socioeconómicos del cambio climático, en la tercera se describen la estructura y el funcionamiento del modelo ECLAC-CIAM. En la cuarta sección se presenta un ejercicio de simulación con el que se analizan los efectos económicos del cambio climático en el año 2050 y en la quinta y última sección se detallan las conclusiones.

\section{II}

\section{El estado de la técnica}

\section{Modelos económicos aplicados para la evaluación del cambio climático}

Para evaluar los efectos económicos del cambio del clima y las políticas en esa materia se han creado varios modelos numéricos aplicados, que difieren en alcance, metodología, nivel de agregación, tratamiento de la tecnología y en la incertidumbre y grado de integración con los modelos climáticos.

En primer lugar, es posible distinguir entre modelos de simulación (positivos) y optimización (normativos). Los modelos de simulación se utilizan para realizar experimentos de tipo “¿qué pasaría si...?”, teniendo en cuenta variaciones en el clima, la política o en ambos. Casi todos los sistemas basados en un modelo de equilibrio general computable (EGC), incluido el ECLAC-CIAM presentado en este trabajo, constituyen modelos de simulación. Entre los modelos de este tipo cabe mencionar los siguientes: DART (Dynamic Applied Regional Trade) (Springer, 1998), ENVISAGE (Roson y van der Mensbrugghe, 2012), EPPA (Emissions Prediction and Policy Analysis) (Paltsev y otros, 2005), GEMINI-E3 (Bernard y Vielle, 2008), GREEN (General Equilibrium Environment Model) (Burniaux, Nicoletti y Oliveira Martins, 1992; Lee, van der Mensbrugghe y Oliveira Martins, 1994), GTAP-E (Burniaux y Troung, 2002), GTEM (Global Trade and Environment Model) (Pant, 2007) e ICES (Intertemporal Computable Equilibrium System) (Eboli, Parrado y Roson, 2010). A diferencia de estos, en los modelos de optimización se toma un objetivo, expresado en una función que ha de maximizarse o minimizarse. Todos los modelos basados en la maximización de la utilidad intertemporal "a la Ramsey" corresponden a esta categoría. Tal vez los más conocidos son los que forman el conjunto de modelos Dice y Rice, elaborados por William Nordhaus (Nordhaus, 1994; Nordhaus y Yang, 1996). Cabe mencionar también los modelos EDGE (Endogenous Technological Change) (Jensen y Thelle, 2001), ENTICE (Popp, 2003), FUND (Anthoff y Tol, 2008), MERGE (Manne, Mendelsohn y Richels, 1995), PAGE (Hope, Anderson y Wenman, 1993), wiagem (Kemfert, 2002) y witch (Bosetti y otros, 2006).

En general, los modelos de simulación son modelos a gran escala, detallados desde una visión regional e industrial. Desde un punto de vista matemático, son grandes sistemas de ecuaciones no lineales, que deben resolverse con paquetes matemáticos generales o un programa computacional especializado (entre ellos, GEMPACK, GAMS/PATH). Por otra parte, los modelos de optimización generalmente son modelos muy agregados, que se resuelven mediante algoritmos de programación no lineal (por ejemplo, GAMS/MINOS, GAMS/CONOPT). En los modelos Dice y Rice, por ejemplo, se tiene en cuenta simplemente un bien agregado que puede utilizarse para consumo o inversión. Los efectos del cambio climático también se modelan de una manera bastante rudimentaria, utilizando una única función de daño que da como resultado una reducción de los posibles ingresos a medida que aumenta la temperatura.

Los diversos enfoques difieren en términos de integración con los modelos climáticos. Algunos enfoques tienen "enlaces simbólicos o blandos" con dichos modelos. Esto significa que un modelo climático (por ejemplo, un Modelo de Circulación Mundial) se utiliza en primer 
lugar para producir un escenario del clima, que se toma como dado dentro del modelo económico. Los resultados de este último pueden entonces introducirse en dicho escenario en un proceso iterativo. Otros modelos tienen "enlaces duros", es decir, que poseen un módulo climático completamente integrado al sistema. La mayoría de los modelos de optimización tienen esta característica, al igual que algunos de simulación a gran escala, como EPPA y ENVISAGE. La ventaja de tener bloques económicos y climáticos coherentes dentro del mismo modelo debe evaluarse en función de la pérdida de complejidad del componente climático, que generalmente es un modelo de circulación general reducido, que incluye solo un número limitado de ecuaciones.

Al utilizar los modelos para analizar el futuro distante se deben tener en cuenta los cambios en la tecnología disponible. La inversión en tecnologías verdes podría promoverse mediante incentivos económicos, posiblemente como parte de un paquete de políticas sobre el clima. Solo en pocos modelos (como WITCH y ENTICE) se aborda explícitamente el tema del "cambio técnico endógeno". Lamentablemente, la estimación de los parámetros es muy difícil, pues en cierto modo es arbitraria y subjetiva. En otros modelos se tiene en cuenta la existencia de "tecnologías de contención", es decir, tecnologías disponibles que son demasiado costosas para ser viables en las condiciones económicas actuales, pero que pueden poner un límite máximo al costo de tecnologías más tradicionales, como las basadas en los combustibles fósiles, que podrían volverse mucho más caras en el futuro.

En el caso de los modelos de optimización intertemporal, entre ellos Dice, Rice y PAGE, se plantea un problema especial, pues requieren el uso de factores de descuento que no pueden estimarse fácilmente a partir de las tasas de interés actuales, cuando el horizonte de optimización es en un futuro lejano y el rendimiento de la inversión resulta afectado por varias incertidumbres. La publicación de The Economics of Climate Change: The Stern Review (Stern, 2007) provocó un intenso debate, en el que se subrayó la extrema sensibilidad de los resultados de estos modelos a las suposiciones sobre los factores de descuento. Asimismo, no existe una única metodología científica correcta que debería seguirse en el proceso de estimación, pues las suposiciones sobre los factores de descuento resultan afectadas por juicios de valor subjetivos (a veces ocultos).

\section{Ventajas y limitaciones de los modelos de EGC para la evaluación de los efectos del cambio climático}

El ECLAC-CIAM es un modelo de equilibrio general computable (EGC) basado en la formulación GTAP, que se amplió para incluir un conjunto de funciones de daño sectorial. Como tal, comparte todas las ventajas y desventajas de otros modelos de equilibrio general en este campo, que presentan las características clave resumidas anteriormente.

Desde una óptica positiva, el elevado nivel de desagregación permite comprender las complejas interdependencias estructurales de los sistemas económicos globalizados y abre el camino a una delimitación precisa de los efectos del cambio climático a una escala industrial y geográfica detallada. Debido a que los modelos de EGC son herramientas de uso general que no se crearon originalmente para analizar el cambio climático, es fácil evaluar la manera en que las políticas en otros ámbitos podrían afectar al clima y viceversa. Los abundantes resultados de los ejercicios de simulación pueden continuar procesándose (como de hecho ocurre en el ECLAC-CIAM). Por ejemplo, es posible estimar el comercio de derechos de emisión a partir de las matrices de origen y destino de los flujos comerciales (Atkinsons y otros, 2010), que constituye una información muy útil en el contexto de las negociaciones internacionales sobre el clima.

Desde un punto de vista negativo, los modelos de EGC exigen una gran cantidad de datos, pues se concibieron originalmente para el análisis de políticas a corto plazo. Su uso con un horizonte temporal mucho mayor puede ser problemático si se registran cambios significativos en la tecnología o las preferencias de los consumidores que modifiquen sustancialmente la estructura económica con respecto a su estado actual. Solo por este motivo no deberían considerarse como herramientas de previsión, ni utilizarse para elaborar hipótesis económicas futuras.

\section{Evaluación del cambio climático en el Caribe}

Básicamente, todos los estudios recientes sobre los efectos del cambio climático en el Caribe se han realizado con el apoyo y bajo los auspicios de la CEPAL, con frecuencia en asociación con otros organismos internacionales. La publicación más reciente se titula "The Economics of Climate Change in the Caribbean - Summary Report 
2011" (CEPAL, 2011). Otros hallazgos del trabajo de investigación aún no publicados se han presentado en seminarios internacionales.

En el citado informe de 2011 se presentan los resultados de distintas hipótesis de cambio climático en el Caribe, obtenidas mediante el empleo de un modelo de circulación regional basado en dos modelos de circulación generales (ECHAM4 y HADCM3). El modelo predice un aumento de 1,78 grados centígrados de la temperatura media de la región en el año 2050 con respecto al punto de referencia de 1960-1990, de acuerdo con el escenario A2 del Informe especial de escenarios de emisiones del Grupo Intergubernamental de Expertos sobre el Cambio Climático (IPCC) (IPCC, 2000), y de 1,84 grados centígrados de acuerdo con el escenario B2 del mismo informe. Los resultados relativos a las precipitaciones son mixtos, con aumentos en algunos países y disminuciones en otros. También se determinó la probabilidad de un incremento de la intensidad de los ciclones tropicales en caso de que las temperaturas continúen en alza.

En el informe también se incluyen varios estudios de impacto sectorial relativos a la agricultura, el ambiente costero y marino, la salud humana, el turismo, el transporte, los recursos hídricos y la energía. Si bien estos estudios no son comparables, tienen el mérito de concentrarse en el Caribe y destacar las dificultades y los problemas para estimar las consecuencias físicas del cambio climático en la región.

\section{III}

\section{Estructura del modelo}

\section{Panorama general}

Como se puede apreciar en el gráfico 1, el modelo ECLAC-CIAM incluye tres módulos que funcionan en forma secuencial.

El primer módulo se utiliza para traducir los valores de las variables climáticas en cambios en los parámetros económicos del componente macroeconómico mundial. El módulo de EGC se utiliza entonces para realizar una simulación estática comparativa y confrontar el estado de la economía mundial antes y después de la variación en los parámetros relativos al clima. En el EGC se tienen en cuenta las siguientes subregiones y países: América del Norte, América del Sur, Centroamérica, el Caribe, Belice,

GRÁFICO 1

Estructura modular del modelo ECLAC-CIAM

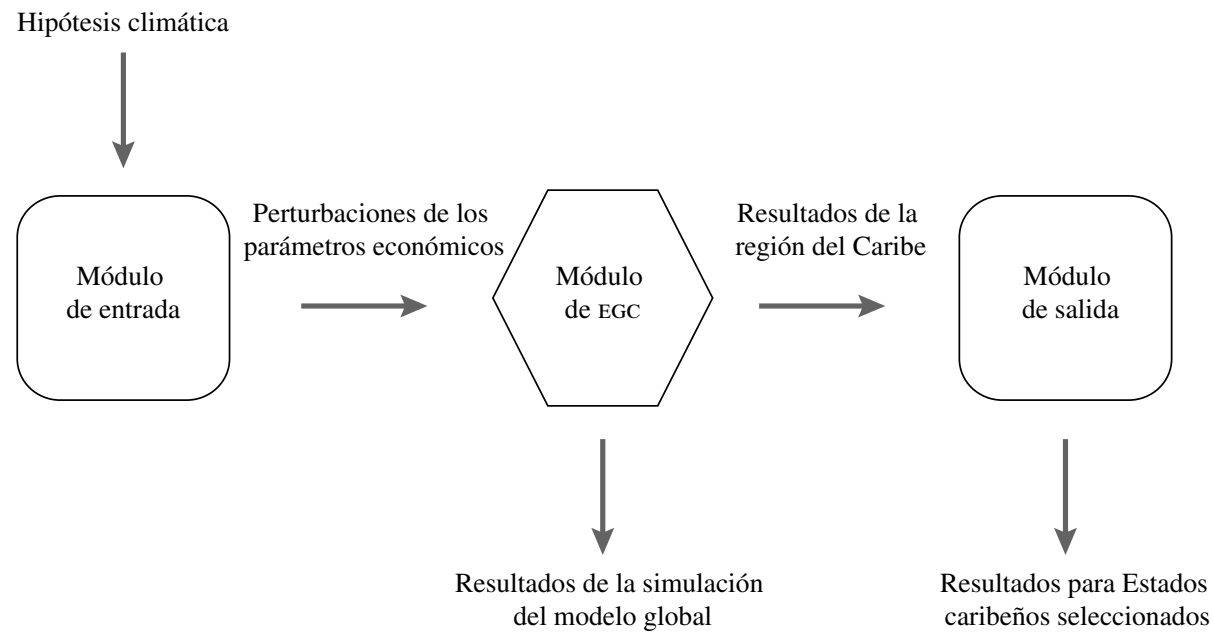

Fuente: elaboración propia.

EGC: equilibrio general computable. 
Guyana y Suriname (considerados en forma conjunta), Europa, África, Asia y Oceanía. Para lograr un mayor detalle geográfico con respecto a la subregión caribeña, se procesan posteriormente los resultados del módulo de EGC y se obtienen los valores aproximados de algunas variables macroeconómicas (en particular, los cambios en el ingreso nacional real) de Estados seleccionados para este estudio. A continuación se describe el funcionamiento de cada módulo en forma más específica.

\section{Funciones de daño sectorial}

El primer módulo del ECLAC-CIAM se utiliza para producir perturbaciones exógenas en diversas variables y parámetros económicos en el modelo de EGC principal, sobre la base de un determinado escenario climático. Esto se realiza mediante la aplicación de "funciones de daño" sectorial.

La función de daño es la relación entre una variable que describe el estado del clima (en este caso, el cambio en la temperatura absoluta de la superficie mundial en grados centígrados desde el año 2000) y algunos parámetros del modelo económico (generalmente expresados como un cambio porcentual con respecto al nivel de referencia). El tipo de impacto sectorial determina los parámetros que se tienen en cuenta. Por ejemplo, los cambios estimados en la productividad agrícola se traducen en cambios porcentuales en el parámetro de productividad multifactorial de la "agricultura" en el modelo de EGC. Los efectos en la salud humana se interpretan como cambios en la dotación o la productividad de la mano de obra, y así sucesivamente.

En el gráfico 2, por ejemplo, se trazan cuatro funciones de daño del sector agrícola obtenidas a partir del modelo de ECG, las que corresponden a las subregiones de Centroamérica, el Caribe y América del Sur y a los países de Suriname y Guyana (considerados en forma conjunta) y Belice. En el eje horizontal se mide el cambio en la temperatura (en grados centígrados) desde el año 2000. En el eje vertical se mide el cambio porcentual estimado en la productividad agrícola en los tres países. Cabe señalar que las estimaciones no se refieren a un cultivo específico, sino al sector agrícola en su totalidad y no tienen en cuenta los cambios en el suministro de agua ni eventuales acontecimientos extremos. La relación entre la temperatura y la productividad en la agricultura no es lineal: un aumento moderado de la temperatura (y de la concentración de dióxido de carbono) es beneficioso, mientras que los niveles de temperatura más altos reducen la productividad agrícola.

GRÁFICO 2

América Latina y el Caribe (países y subregiones seleccionados): cinco funciones de daño (agricultura)

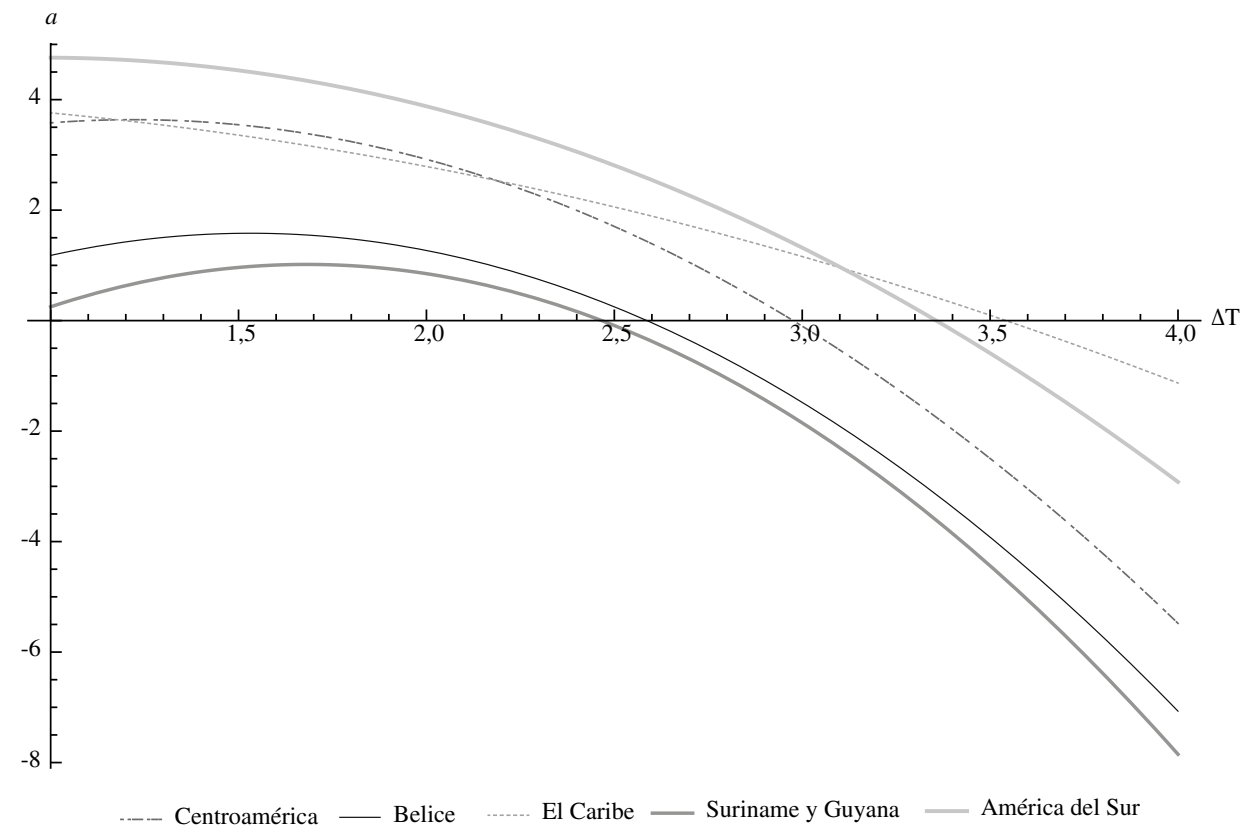

Fuente: elaboración propia. 
Otras funciones de daño en el ECLAC-CIAM representan relaciones lineales simples, con frecuencia debido a la falta de información suficiente para estimar los diversos parámetros de funciones no lineales. Por ejemplo, en el gráfico 3 se trazan cinco funciones de daño para el efecto del aumento del nivel del mar. En este caso, en el eje vertical se mide la pérdida porcentual en dotación de capital y tierras en cada país. Resulta evidente que los pequeños Estados insulares del Caribe son muy vulnerables al incremento del nivel del mar.

El ECLAC-CIAM comprende siete efectos sectoriales, y cada uno de ellos está vinculado a una función de daño específica. Además de la agricultura y el aumento del nivel del mar, se tienen en cuenta los siguientes efectos:

- Disponibilidad de agua: esta es una segunda fuente de variación de la productividad agrícola, que se asume dependiente de cambios estimados en la escorrentía. De acuerdo con los valores de los parámetros actuales, un aumento de la temperatura de 1 grado centígrado reduciría la productividad agrícola en el Caribe un 0,21\% debido a la menor disponibilidad de agua.

- Turismo: los cambios en la temperatura se relacionan con cambios en los ingresos netos por concepto de turismo desde el extranjero, que corresponden a transferencias de ingresos extranjeros en el modelo de EGC. Un aumento de la temperatura de 1 grado centígrado reduciría los ingresos del turismo en 8.600 millones de dólares en Centroamérica, 10 millones de dólares en Belice, 5.500 millones de dólares en el Caribe y 204 millones de dólares en Suriname y Guyana, pero acrecentaría los ingresos del turismo en 26.800 millones de dólares en América del Sur.

- Demanda de energía: se refiere a cambios en el consumo de energía de los hogares según las necesidades de refrigeración y calefacción. En el modelo de EGC, las variaciones en el consumo de energía se ajustan mediante cambios en todos los demás gastos, de modo que la restricción presupuestaria para cada hogar de cada país se mantiene. Un aumento de la temperatura de 1 grado centígrado incrementaría el consumo de energía un $0,21 \%$ en Belice y un $0,24 \%$ en el Caribe, mientras que lo reduciría un $0,23 \%$ en Centroamérica, un $0,25 \%$ en Suriname y Guyana, y un $0,02 \%$ en América del Sur.

- Salud humana: los casos adicionales (mortalidad y morbilidad) de enfermedades relacionadas con el frío, el calor y transmitidas por vectores se traducen en cambios en la productividad del trabajo en cada

GRÁFICO 3

América Latina y el Caribe (países y subregiones seleccionados): cinco funciones de daño (aumento del nivel del mar)

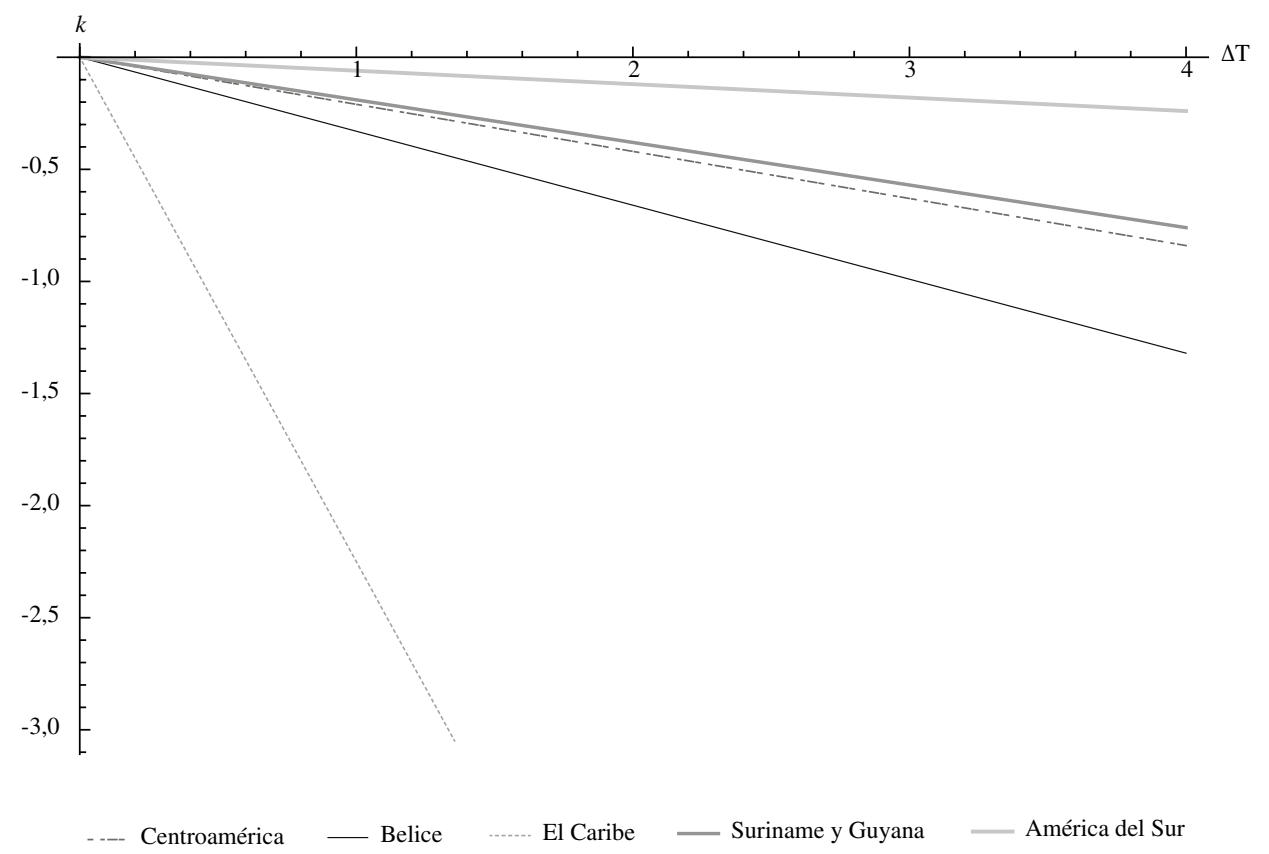

Fuente: elaboración propia. 
región. Un aumento de la temperatura de 1 grado centígrado reduciría la productividad del trabajo un 0,58\% en Centroamérica, un 0,57\% en Belice, un $0,13 \%$ en el Caribe y un $0,11 \%$ en Suriname y Guyana y en el resto de América del Sur.

- Productividad en el trabajo: se refiere a la productividad del trabajo en actividades al aire libre, que puede depender directamente de la temperatura y la humedad. Un aumento de la temperatura de 1 grado centígrado disminuiría la productividad del trabajo (media) un $0,43 \%$ en las cinco regiones y países de referencia, excepto en América del Sur donde se reduciría un $0,38 \%$.

Todas estas cifras son indicativas y deberían tratarse con precaución. Los parámetros de las funciones de daño pueden cambiarse a medida que se dispone de información más confiable. En la actualidad, los valores de los parámetros se calculan sobre la base de una gran variedad de estudios sectoriales y corresponden a los valores empleados en el modelo de evaluación integrado ENVISAGE (Roson y van der Mensbrugghe, 2012), elaborado en el Banco Mundial.

En la mayoría de los estudios sectoriales utilizados para este propósito no se hace una referencia explícita al Caribe u otras regiones tropicales. Por ejemplo, los parámetros de las funciones de daño de la productividad agrícola (véase el gráfico 2) se obtuvieron a partir del promedio ponderado de las funciones de respuesta de tres cultivos, a saber: trigo, arroz y maíz. Si bien se trata de los tres cultivos más difundidos en todo el mundo, ciertamente no son representativos de la agricultura tropical. Se espera que para las futuras versiones del ECLAC-CIAM se disponga de datos y estimaciones de parámetros mejores y más confiables.

\section{El modelo de EGC principal}

El núcleo del ECLAC-CIAM es un modelo de equilibrio general computable GTAP estándar. Se trata de un modelo macroeconómico de gran medida que incluye miles de ecuaciones agrupadas en 213 categorías. Su descripción formal completa excede el objetivo de este artículo, pero puede encontrarse fácilmente en Hertel y Tsigas (1997). El modelo de EGC, que no fue diseñado específicamente para el análisis del cambio climático, ofrece una representación de la economía mundial y sus interdependencias entre regiones, industrias y mercados.

La mayoría de los parámetros del modelo de EGC están calibrados. Esto significa que sus valores están establecidos de manera tal que el punto de referencia del modelo reproduce exactamente los datos estadísticos observados, como los niveles de consumo y los patrones comerciales. Los ejercicios de simulación se realizan mediante la modificación de las variables exógenas (como las tasas de impuestos, los factores de productividad y la dotación de factores, entre otras) y el cálculo de un equilibrio general contrafactual de la economía mundial en que los mercados de productos y factores primarios invariablemente se equilibran. En consecuencia, más que para predecir escenarios económicos futuros, los modelos de EGC están diseñados principalmente para estudiar los procesos de ajuste estructural provocados por los cambios en algunos parámetros.

La producción en cada industria regional se realiza mediante el empleo de factores primarios e intermedios. Los factores intermedios se producen en otras industrias, nacionales o extranjeras. El papel de cada factor en el proceso de producción se determina al calibrar el modelo, es decir, cuando se fijan los valores de los parámetros de acuerdo con las estructuras de costos industriales observadas. La demanda de factores de producción puede cambiar en los ejercicios de simulación debido a variaciones en los niveles de producción y en los precios relativos. Los factores costosos se sustituyen (parcialmente) con otros menos caros, sobre la base de las funciones de producción industrial y los parámetros de "elasticidad de sustitución" asumidos (que determinan el grado de sensibilidad de los patrones de los factores a los precios relativos).

Debido a que las industrias regionales son grandes conjuntos heterogéneos, los bienes producidos en la misma industria, pero en diferentes regiones, se consideran como bienes distintos. La demanda intermedia y final de los productos se divide en una estructura de anidamiento: en primer lugar, los precios relativos determinan la cantidad de importaciones y el tamaño de la demanda interna; en segundo término, las importaciones se distribuyen entre diferentes fuentes extranjeras, una vez más sobre la base de los precios relativos y la elasticidad de sustitución (que puede variar por región y sector). Los precios de los bienes importados incluyen márgenes comerciales y transporte internacional, aranceles y barreras no arancelarias.

En un estado de equilibrio, los volúmenes de producción de cada industria regional deben coincidir con la demanda total, que comprende la demanda intermedia de otras industrias nacionales y extranjeras, el consumo de los hogares nacionales y extranjeros, el gasto público y la demanda nacional y extranjera de inversión (física). Las condiciones de equilibrio se logran mediante el establecimiento de precios adecuados para los productos y los factores primarios. 
Si bien la dotación de recursos primarios generalmente ya está dada, es posible cambiar la división entre las variables endógenas y exógenas del modelo. Los factores primarios son inmóviles a nivel internacional, pero total o parcialmente móviles entre sectores de la economía nacional. En un estado de equilibrio, la dotación de factores debe coincidir con la demanda generada por las diversas industrias nacionales.

El ingreso nacional es el valor de todos los recursos primarios de propiedad nacional. Esto incluye salarios, rendimientos de capital, rentas de recursos y tierras e ingresos fiscales. El ingreso nacional se distribuye entre el consumo de hogares privados, el gasto público y los ahorros. Los patrones de consumo se determinan sobre la base de la maximización de la función de utilidad sujeta a restricciones presupuestarias. En consecuencia, la demanda de consumo final es sensible a los precios relativos.

Los ahorros regionales se colocan hipotéticamente en un banco internacional virtual, que luego los destina a inversiones regionales sobre la base del rendimiento futuro esperado (vinculado con el rendimiento actual). Como consecuencia, los ahorros y las inversiones regionales no necesariamente coinciden. Las identidades contables nacionales suponen que los ahorros excedentes reflejan un superávit del comercio exterior y viceversa. Sin embargo, las condiciones de equilibrio requieren que la balanza de pagos, en lo posible incluidas las transferencias y remesas extranjeras, sea igual a cero. Esta condición se satisface adaptando los tipos de cambio internacionales.

Cuando el modelo está calibrado, los valores de los parámetros se fijan de modo que los niveles de producción, consumo e inversión se calculan en forma endógena, conforme con las estadísticas de las cuentas nacionales. Los equilibrios contrafactuales se obtienen cambiando los valores de calibración de las variables y los parámetros endógenos.

Esto es exactamente lo que ocurre al realizar un ejercicio de simulación con el ECLAC-CIAM. Las funciones de daño del cambio climático se utilizan en primer lugar para analizar la manera en que las variaciones de las condiciones climáticas (cambios en la temperatura media de la superficie) afectan a cierto número de variables exógenas en el modelo de EGC, como la productividad del trabajo, la productividad agrícola total, la dotación de tierra y capital, y así sucesivamente. Todos los cambios en las variables exógenas se ingresan simultáneamente en el modelo de EGC, que luego se utiliza para calcular niveles constantes de variables como precios relativos, niveles de ingreso y utilidad, inversiones, patrones de comercio internacional, volúmenes de producción e ingresos fiscales, entre otros. Esta amplia serie de resultados ofrece un panorama general de las consecuencias económicas del cambio climático.

\section{Reducción de escala}

Si bien los resultados de la simulación de un modelo de EGC pueden ser cuantiosos, en algunas circunstancias es factible que no alcancen el nivel de detalle deseado. Por ejemplo, no es posible conocer directamente los efectos del cambio climático en Estados específicos de la subregión del Caribe, pues la calibración del modelo de EGC requiere datos contables nacionales muy detallados que no están disponibles.

No obstante, algunos resultados pueden obtenerse de manera indirecta mediante el empleo de técnicas de microsimulación. En el ECLAC-CIAM, estas técnicas se utilizan para obtener estimaciones indirectas de cambios en los ingresos reales, es decir, en el producto interno bruto (PIB) de los Estados insulares del Caribe seleccionados para este análisis; ellos son:

- Antigua y Barbuda

- Barbados

- Bermudas

- Pequeños Estados insulares del Caribe

- Cuba

- Dominica

- República Dominicana

- Granada

- Jamaica

- Saint Kitts y Nevis

- Santa Lucía

- San Vicente y las Granadinas

- Trinidad y Tabago

Esto es posible porque el PIB es simplemente la suma del valor agregado producido por todas las industrias de un país. Además de estimaciones del PIB (real y nominal) de todas las regiones del conjunto, el modelo de EGC proporciona estimaciones de cambios en el valor agregado industrial en todas ellas, incluido el Caribe. En la base de datos de los Indicadores del Desarrollo Mundial del Banco Mundial se puede acceder a la composición sectorial del ingreso nacional de todos los países anteriormente mencionados y de las seis industrias del modelo de EGC. Por consiguiente, es posible obtener una aproximación razonable al cambio en el ingreso nacional calculando un promedio ponderado de los cambios en el valor agregado industrial, en que los pesos están dados por las proporciones sectoriales del PIB nacional. Esta operación se realiza en el módulo de salida del ECLAC-CIAM. 


\section{Requisitos de datos}

La aplicación del ECLAC-CIAM requiere datos para implementar el modelo de EGC e información para estimar los parámetros de las funciones de daño y realizar el análisis posterior al procesamiento. Los parámetros de las funciones de daño se obtuvieron en distintos estudios en los que se examinan diversos efectos del cambio climático. El procedimiento de reducción de escala se basa en datos relativos a la composición del valor agregado del PIB nacional en los Estados caribeños.

Si bien las elasticidades de sustitución constituyen una excepción, muchos de los parámetros de los modelos de EGC están calibrados, pues se toman de la literatura o de valores de referencia convencionales. La calibración de un modelo de EGC supone la elección de valores de parámetros, de modo que el modelo calcule en forma endógena el volumen de los flujos comerciales, el consumo y la producción en forma coherente con las matrices de contabilidad social o de insumo-producto disponibles. Si bien el proceso de calibración puede ser largo y engorroso (y depende de la disponibilidad de los datos), en la actualidad se dispone de recursos de datos estandarizados y globales, lo que facilita mucho las cosas. El ECLAC-CIAM se ha calibrado utilizando la Base de datos GTAP 8.

Desde 1992, el consorcio GTAP (Global Trade Analysis Project), encabezado por la Universidad de
Purdue de los Estados Unidos de América, recoge información sobre las cuentas nacionales con el objetivo de construir y mantener una base de datos matriz de contabilidad social mundial. La base de datos GTAP 8 es la octava edición pública de la Base de datos GTAP desde el comienzo del proyecto. Las bases de datos GTAP se crean a partir de las bases de datos o cuadros de insumo-producto nacionales, que se combinan con otros conjuntos de datos internacionales sobre agregados macroeconómicos, comercio bilateral, energía, insumoproducto agrícola y protección comercial para los nuevos años de referencia. A medida que se dispone de conjuntos importantes de datos actualizados se construyen versiones provisorias de la base de datos. También se realizan mejoras en los procedimientos de obtención, aplicación y construcción de datos. En la GTAP 8 los datos proceden de una gran variedad de fuentes, incluidos el Banco Mundial, organismos nacionales de estadística, el Centro de Comercio Internacional/ Centro de información y estudios prospectivos internacionales, la Base de datos estadísticos sobre el comercio de mercaderías (COMTRADE por sus siglas en inglés), la Agencia Internacional de la Energía (AIE), la Organización de Cooperación y Desarrollo Económicos (OCDE) y muchas otras, y se concilian en un marco coherente. El proceso de construcción es bastante complejo y se documenta en forma exhaustiva en www.gtap.org.

\section{IV}

\section{Ejemplo de ejercicio de simulación}

Para ilustrar el funcionamiento del ECLAC-CIAM y el tipo de resultados que puede producir, en esta sección se presenta un ejemplo de ejercicio de simulación ${ }^{1}$. Para ello se examinará el escenario A2 del Informe especial de escenarios de emisiones del Grupo Intergubernamental de Expertos sobre el Cambio Climático para el año 2050, que supone un aumento de la temperatura media de 1,2 grados centígrados con respecto al año 2000. Las funciones de daño expresan esta hipótesis en términos de cambios en las siguientes variables económicas:

- un aumento de la productividad de la tierra del $3,38 \%$ en Centroamérica, de $1,03 \%$ en Belice,

\footnotetext{
${ }^{1}$ Los lectores interesados pueden reproducir fácilmente este ejercicio.
}

de $0,68 \%$ en el Caribe, de $0,41 \%$ en Suriname y Guyana y de 4,64\% en América del Sur;

- una reducción del capital de $0,25 \%$ en Centroamérica, de $0,40 \%$ en Belice, de 2,69\% en el Caribe, de 0,22\% en Suriname y Guyana y de $0,08 \%$ en América del Sur;

- una disminución de la productividad del trabajo de $1,10 \%$ en Centroamérica, de $1,20 \%$ en Belice, de $0,67 \%$ en el Caribe, de $0,64 \%$ en Suriname y Guyana y de $0,58 \%$ en América del Sur;

- una contracción de la demanda de servicios turísticos del 1,38\% en Centroamérica, de 1,43\% en Belice, de 3,34\% en el Caribe y de 7,52\% en Suriname y Guyana, y un aumento del 2,22\% en América del Sur, 
- una merma de la producción de energía de $0,05 \%$ en Belice, Suriname y Guyana, de $0,06 \%$ en el Caribe y Centroamérica y de $0,04 \%$ en América del Sur.

Estos, al igual que los valores correspondientes a todas las otras regiones, constituyen perturbaciones exógenas para el modelo de equilibrio general. Una vez ejecutado el modelo de EGC se vuelven disponibles las estimaciones relativas a diversas variables económicas. Por ejemplo, en el cuadro 1 se detallan los cambios estimados en los volúmenes de producción en los sectores de la agricultura, la energía, la industria ligera, la industria pesada, los servicios de mercado y los servicios no de mercado en tres subregiones y tres países de América Latina y el Caribe (si bien en el modelo se dispone de resultados correspondientes a todas las regiones e industrias).

Cabe señalar que el cambio exógeno en la productividad de la tierra no coincide con el cambio estimado en el volumen de la producción agrícola. Un razonamiento similar se aplica a la producción de energía y los servicios de mercado. Esto se debe a que el modelo da cuenta de los cambios en la competitividad relativa y los términos de intercambio. En el Caribe, por ejemplo, la menor demanda de servicios de mercado (como consecuencia del menor atractivo de las metas turísticas) se traduce en volúmenes inferiores de producción en la agricultura (que constituye un proveedor de la industria turística). También supone una devaluación real de la moneda local, que disminuye los costos de producción y favorece la competitividad internacional en los sectores de energía y manufacturero.

En el cuadro 2 se muestran los cambios porcentuales en los niveles de consumo de los hogares por industria. Los niveles más bajos indican una reducción del bienestar. Los cambios en los precios relativos también producen cambios en los patrones de consumo.

Los resultados de la simulación revelan que los efectos del cambio climático pueden dar lugar a una reducción bastante considerable de los niveles de consumo, especialmente en el Caribe. El ECLAC-CIAM también puede proporcionar un análisis más detallado del bienestar, por ejemplo, al calcular la variación equivalente en cada región, que es un índice de medición monetaria de los efectos en el bienestar. La variación equivalente es el cambio en el ingreso que habría producido la misma variación en los niveles de utilidad, a precios constantes. Se concluye que en el año 2050 el cambio climático producirá en

CUADRO 1

América Latina y el Caribe (países y subregiones seleccionados): variaciones en los volúmenes de producción industrial (En porcentajes)

\begin{tabular}{lccccc}
\hline & Centroamérica & Belice & El Caribe & Suriname y Guyana & América del Sur \\
\hline Agricultura & $-0,13$ & $-1,83$ & 0,39 & $-0,6$ & $-1,84$ \\
Energía & 1,21 & 0,09 & 4,1 & 0,79 & $-2,75$ \\
Industria ligera & 0,61 & 0,26 & 2,0 & 3,29 & $-1,24$ \\
Industria pesada & 1,72 & $-0,27$ & 3,71 & 6,57 & $-2,51$ \\
Servicios de mercado & $-1,64$ & $-1,38$ & $-4,22$ & $-7,33$ & 1,67 \\
Servicios no de mercado & $-0,95$ & $-1,1$ & $-1,82$ & $-0,99$ & $-0,69$ \\
\hline
\end{tabular}

Fuente: elaboración propia.

CUADRO 2
América Latina y el Caribe (países y subregiones seleccionados): variaciones en los volúmenes de consumo de los hogares (En porcentajes)

\begin{tabular}{|c|c|c|c|c|c|}
\hline & Centroamérica & Belice & El Caribe & Suriname y Guyana & América del Sur \\
\hline Agricultura & 0,21 & $-0,23$ & $-1,1$ & $-0,64$ & 0,6 \\
\hline Energía & $-0,87$ & $-1,55$ & $-4,12$ & $-1,95$ & 0,17 \\
\hline Industria ligera & $-0,38$ & $-1,16$ & $-2,55$ & $-1,29$ & 0,22 \\
\hline Industria pesada & $-0,89$ & $-1,8$ & $-4,13$ & $-2,16$ & $-0,05$ \\
\hline Servicios de mercado & $-0,72$ & $-1,71$ & $-3,37$ & $-1,91$ & $-0,26$ \\
\hline Servicios no de mercado & $-0,86$ & $-1,91$ & $-3,05$ & $-1,77$ & $-0,35$ \\
\hline
\end{tabular}

Fuente: elaboración propia. 
Centroamérica consecuencias económicas equivalentes a una pérdida de alrededor de 7.500 millones de dólares por año. La variación equivalente de las pérdidas en las otras regiones y países de referencia es de 17 millones de dólares en Belice, 6.700 millones de dólares en el Caribe, 84 millones de dólares en Suriname y Guyana y 4.200 millones de dólares en América del Sur.

La magnitud de la pérdida depende claramente del tamaño de la economía regional. Para apreciar mejor los efectos en el bienestar, en el cuadro 3 se muestra la variación equivalente relativa al ingreso nacional, que representa la variación porcentual en el PIB real. Además de los resultados relativos a las cinco regiones agregadas, se presenta también la variación estimada con respecto a algunos países individuales del Caribe, obtenida a partir del módulo de salida del ECLAC-CIAM, así como otras estimaciones cuyo significado se explica a continuación.

En la columna correspondiente a "Total" se detalla el cambio estimado en el PIB regional. El impacto en el PIB del Caribe es bastante grande: se prevé que en 2050, a causa del cambio climático, el ingreso nacional será alrededor de un $3 \%$ más bajo que su nivel hipotético en ausencia de cambio climático. Los resultados correspondientes a Belice, Guyana y Suriname también son bastante significativos, mientras que el impacto en Centroamérica y América del Sur es bastante menor.

Las columnas "Superior" e "Inferior" se refieren a un análisis de sensibilidad de los resultados de la simulación. En este ejemplo, se informó al programa computacional del modelo que había incertidumbre sobre los valores correctos de algunos parámetros sometidos a perturbaciones. Más específicamente, se asumió que los cambios en la productividad del trabajo y la demanda de servicios de mercado podrían tomar cualquier valor en el intervalo $+/-50 \%$ de la estimación de referencia. En otras palabras, se reemplazó un valor individual por la variación en parámetros específicos con una distribución de probabilidad (rectangular). Mediante el modelo se podía entonces inferir la distribución de probabilidad de las variables de salida vinculadas a la distribución de las perturbaciones de entrada. Esto se obtuvo realizando varias veces la simulación con distintos valores de entrada, mediante la técnica de cuadratura estadística de Stroud (Stroud, 1957; DeVuyst y Preckel, 1997). En consecuencia, no solo se produce información acerca de los valores centrales para todas las variables de salida, sino también sobre otros momentos estadísticos, como la desviación estándar. En el cuadro 3, en la columna "Inferior" se aprecia el cambio estimado en el PIB real, menos su variación estándar estimada, mientras que en la columna "Superior" la variación estándar se suma. Por lo tanto, los dos valores representan los límites de un intervalo de probabilidad del cambio estimado en el PIB regional.

En las cuatro columnas restantes a la derecha se presentan los resultados de otros ejercicios de simulación en los que se tiene en cuenta solo un tipo de efecto del

América Latina y el Caribe (países y subregiones seleccionados): variaciones en el ingreso nacional real

(En porcentajes)

\begin{tabular}{|c|c|c|c|c|c|c|c|}
\hline & Total & Inferior & Superior & Tierra & Trabajo & Capital & Turismo \\
\hline Centroamérica & $-0,73$ & $-0,84$ & $-0,63$ & 0,03 & $-0,43$ & $-0,18$ & $-0,16$ \\
\hline Belice & $-1,46$ & $-1,69$ & $-1,24$ & $-0,33$ & $-0,75$ & $-0,17$ & $-0,22$ \\
\hline El Caribe & $-2,92$ & $-3,47$ & $-2,37$ & 0,04 & $-0,43$ & $-1,49$ & $-1,05$ \\
\hline Antigua y Barbuda & $-3,20$ & $-3,72$ & $-2,74$ & $-0,09$ & $-0,43$ & $-1,51$ & $-1,24$ \\
\hline Barbados & $-3,11$ & $-3,64$ & $-2,61$ & $-0,06$ & $-0,43$ & $-1,50$ & $-1,18$ \\
\hline Bermudas & $-3,76$ & $-4,17$ & $-3,51$ & $-0,11$ & $-0,46$ & $-1,54$ & $-1,55$ \\
\hline Pequeños Estados insulares del Caribe & $-2,71$ & $-3,31$ & $-2,06$ & $-0,03$ & $-0,42$ & $-1,48$ & $-0,96$ \\
\hline Cuba & $-3,22$ & $-3,71$ & $-2,77$ & 0,03 & $-0,44$ & $-1,51$ & $-1,21$ \\
\hline Dominica & $-3,20$ & $-3,67$ & $-2,80$ & 0,25 & $-0,45$ & $-1,50$ & $-1,13$ \\
\hline República Dominicana & $-2,68$ & $-3,28$ & $-2,04$ & 0,05 & $-0,42$ & $-1,48$ & $-0,92$ \\
\hline Granada & $-3,15$ & $-3,66$ & $-2,67$ & $-0,02$ & $-0,43$ & $-1,51$ & $-1,19$ \\
\hline Jamaica & $-2,97$ & $-3,52$ & $-2,43$ & 0,01 & $-0,43$ & $-1,49$ & $-1,09$ \\
\hline Saint Kitts y Nevis & $-3,17$ & $-3,69$ & $-2,68$ & $-0,10$ & $-0,43$ & $-1,51$ & $-1,23$ \\
\hline Santa Lucía & $-3,29$ & $-3,78$ & $-2,87$ & $-0,04$ & $-0,44$ & $-1,51$ & $-1,27$ \\
\hline San Vicente y las Granadinas & $-3,14$ & $-3,65$ & $-2,67$ & 0,05 & $-0,44$ & $-1,50$ & $-1,16$ \\
\hline Trinidad y Tabago & $-1,61$ & $-2,45$ & $-0,52$ & $-0,17$ & $-0,37$ & $-1,42$ & $-0,42$ \\
\hline Suriname y Guyana & $-1,66$ & $-2,05$ & $-1,27$ & $-0,08$ & $-0,40$ & $-0,12$ & $-1,06$ \\
\hline América del Sur & $-0,19$ & $-0,30$ & $-0,08$ & $-0,01$ & $-0,36$ & $-0,04$ & 0,22 \\
\hline
\end{tabular}

Fuente: elaboración propia. 
cambio climático a la vez. En la columna "Trabajo", por ejemplo, las estimaciones de las variaciones en el PIB real se obtienen modificando solo los parámetros de productividad del trabajo, mientras que todos los demás parámetros (como por ejemplo, la productividad de la tierra) se mantienen invariables. De esta manera, es posible determinar la contribución de un efecto específico del cambio climático al resultado general. Se

\section{V}

\section{Conclusiones}

El ECLAC-CIAM es una plataforma de modelado creada para evaluar las consecuencias económicas del cambio climático en el Caribe. Con este modelo se procura colmar la brecha de conocimientos al permitir la evaluación cuantitativa de los efectos económicos del cambio del clima.

Si bien puede mejorarse de varias maneras (con datos económicos de mejor calidad, estimaciones más confiables de los efectos físicos directos del cambio del clima, escenarios climáticos mejorados, mayor desagregación industrial y regional, entre otros), la versión actual del modelo ECLAC-CIAM, que puede consultarse, descargarse e incluso modificarse libremente, es un modelo de vanguardia totalmente funcional.

Como tal, puede utilizarse fácilmente para realizar ejercicios de simulación como el presentado en este trabajo. A partir de esta sencilla simulación ilustrativa se pueden obtener varios datos clave. Los resultados confirman que el Caribe es una región muy vulnerable en la que el cambio climático producirá consecuencias económicas considerables y negativas. El aumento del nivel del mar, que se traducirá en la pérdida de tierras e infraestructura de capital, y la contracción en la industria puede observar que las variaciones en la productividad del trabajo son de hecho el efecto económico más importante del cambio climático en Belice, Centroamérica y América del Sur. Las pérdidas de capital vinculadas al aumento del nivel del mar constituyen el impacto más importante en el Caribe, mientras que una disminución de la demanda del turismo es el factor más importante en Suriname y Guyana. del turismo constituyen los dos factores más importantes. Estos resultados pueden contribuir a la formulación de políticas de mitigación y adaptación, tanto a nivel regional como internacional.

El modelo es muy flexible y se pueden diseñar fácilmente otros ejercicios de simulación, por ejemplo, con variaciones en el horizonte temporal, el escenario climático o los efectos sectoriales. Los demás parámetros exógenos del modelo de EGC también pueden modificarse, lo que resulta particularmente útil para estudiar las interacciones entre el clima y otras políticas (reformas fiscales, liberalización comercial y crecimiento de la productividad, entre otras).

También es posible "intercambiar" variables endógenas y exógenas en el modelo. Por ejemplo, en el cierre estándar mediante el modelo se calculan los salarios en equilibrio para asegurar el pleno empleo de la mano de obra dada. $\mathrm{Si}$, en cambio, los salarios (reales) se especifican ex ante, la demanda de trabajo puede volverse endógena. La diferencia entre la oferta de trabajo total y la demanda estimada se interpreta de inmediato como desempleo involuntario. 


\section{Bibliografía}

Anthoff, D. y R.S.J. Tol (2008), "The impact of climate change on the balanced-growth-equivalent”, Papers, $\mathrm{N}^{\circ} 228$, Dublín, Economic and Social Research Institute.

Atkinson, G. y otros (2010), "Trade in 'virtual carbon': empirical results and implications for policy", Policy Research Working Paper Series, $\mathrm{N}^{\circ}$ 5194, Washington, D.C., Banco Mundial.

Bernard, A. y M. Vielle (2008), "GEMINI-E3, a general equilibrium model of international-national interactions between economy, energy and the environment", Computational Management Science, vol. 5, $\mathrm{N}^{\circ} 3$, Springer.

Bosetti, V. y otros (2006), "WITCH: a world induced technical change hybrid model", The Energy Journal, vol. 27, Cleveland, International Association for Energy Economics.

Burniaux, J.-M., G.. Nicoletti y J. Oliveira Martins (1992), “GREEN: a global model for quantifying the costs of policies to curb $\mathrm{CO}_{2}$ emissions", OECD Economic Studies, $\mathrm{N}^{\circ} 19$, París, Organización de Cooperación y Desarrollo Económicos (OCDE).

Burniaux, J.-M. y T.P. Truong (2002), "GTAP-E: An energyenvironmental version of the GTAP model", GTAP Technical Paper, $\mathrm{N}^{\circ} 16$, West Lafayette, Indiana.

CEPAL (Comisión Económica para América Latina y el Caribe) (2011), The Economics of Climate Change in the Caribbean: Summary Report 2011 (LC/CAR/L.299), Puerto España, sede subregional de la CEPAL para el Caribe, septiembre.

DeVuyst, E.A. y P.V. Preckel (1997), "Sensitivity analysis revisited: a quadrature-based approach", Journal of Policy Modelling, vol. 19, $\mathrm{N}^{\circ}$ 2, Amsterdam, Elsevier.

Eboli, F., R. Parrado y R. Roson (2010), "Climate change feedback on economic growth: explorations with a dynamic general equilibrium model", Environment and Development Economics, vol. $15, \mathrm{~N}^{\circ} 5$, Cambridge, Cambridge University Press.

Hertel, T.W. y M.E. Tsigas (1997), "Structure of GTAP", Global Trade Analysis: Modeling and Applications, T.W. Hertel (ed.), Cambridge, Cambridge University Press.

Hope, C., J. Anderson y P. Wenman (1993), "Policy analysis of the greenhouse effect: an application of the PAGE model", Energy Policy, vol. 21, $\mathrm{N}^{\circ} 3$, Amsterdam, Elsevier.

IPCC (Grupo Intergubernamental de Expertos sobre el Cambio Climático) (2000), IPCC Special Report Emissions Scenarios, Ginebra.

Jensen, J. y M.H. Thelle (2001), "What are the gains from a multi-gas strategy?", FEEM Working Paper, $N^{\circ} 84$, Milán, Fondazione Eni Enrico Mattei.
Kemfert, C. (2002), "An integrated assessment model of economyenergy-climate - The model WIAGEM", Integrated Assessment, vol. $3, \mathrm{~N}^{\circ} 4$, Routledge.

Lee, H., D. van der Mensbrugghe y J. Oliveira Martins (1994), "The OECD GREEN model: an updated overview", OECD Development Centre Working Papers, $\mathrm{N}^{\circ}$ 97, París, OECD Publishing.

Manne, A.S., M. Mendelsohn y R. Richels (1995), "MERGE - A model for evaluating regional and global effects of GHG reduction policies", Energy Policy, vol. 23, № 1, Amsterdam, Elsevier.

Nordhaus, W.D. (1994), Managing the Global Commons: The Economics of Climate Change, Cambridge, Massachusetts, The MIT Press.

Nordhaus, W.D. y Z. Yang (1996), "A regional dynamic generalequilibrium model of alternative climate-change strategies", American Economic Review, vol. 86, $\mathrm{N}^{\circ} 4$, Nashville, Tennessee, American Economic Association.

Paltsev, S. y otros (2005), "The MIT emissions prediction and policy analysis (EPPA) model: version 4", Report, $\mathrm{N}^{\circ} 125$, Cambridge, Massachusetts, MIT Joint Program on the Science and Policy of Global Change.

Pant, H. (2007), "GTEM: Global Trade and Environment Model”, Technical Report, Canberra, Australian Bureau of Agricultural and Resource Economics and Sciences (ABARES).

Popp, D. (2003), "ENTICE: endogenous technological change in the DICE model of global warming", NBER Working Paper, $\mathrm{N}^{\circ}$ 9762, Cambridge, Massachusetts, National Bureau of Economic Research.

Roson, R. y D. van der Mensbrugghe (2012), "Climate change and economic growth: impacts and interactions", International Journal of Sustainable Economy, vol. 4, $\mathrm{N}^{\circ} 3$, Inderscience Enterprises Ltd.

Springer, K. (1998), "The DART general equilibrium model: a technical description”, Kiel Working Papers, $\mathrm{N}^{\circ} 883$, Kiel, Kiel Institute for the World Economy.

Stern, N. (2007), The Economics of Climate Change: The Stern Review, Cambridge, Cambridge University Press.

Stroud, A.H. (1957), "Remarks on the disposition of points in numerical integration formulas", Mathematical Tables and Other Aids to Computation, vol. 11, $\mathrm{N}^{\circ}$ 60, Providence, American Mathematical Society. 